\title{
Estimulación de motricidad fina con un videojuego en un paciente con hemiparesia izquierda
}

\author{
Fabricio Tipantocta ${ }^{1}$, Flor Tipan ${ }^{1}$, Edison Coral ${ }^{1}$, Gladys Herrera ${ }^{1}$, Geovanny Pacheco ${ }^{1}$ \\ ${ }^{1}$ Carrera en Electrónica Industrial, Instituto Superior Tecnológico Sucre, \\ Teodoro Gómez de la Torre, S14-72. 170148 Quito, Ecuador
}

${ }^{1}$ fftipantocta, ftipan, ecoral, gherrera, gpacheco\}@tecnologicosucre.edu.ec

\section{RESUMEN}

El objetivo del proyecto es realizar un videojuego, adaptando un sistema electrónico en el brazo a un paciente con hemiparesia, y así estimular la motricidad fina en su extremidad superior izquierda lo que aporta a su rehabilitación. Se realizó varios experimentos tomando datos de funcionamiento como son el número de puntos del jugador obtenidos en varias pruebas y se ejecutó el análisis de movimiento en la extremidad afectada tanto en brazo como muñeca. Como resultado se observa el trabajo realizado por el brazo izquierdo del paciente, a través del análisis de video se examina el resultado de flexo extensión de la biomecánica del miembro a rehabilitar, cumpliendo así el objetivo del proyecto. El videojuego al ser amigable por el ambiente de fantasía incentiva el interés del paciente con hemiparesia izquierda, haciendo que a este le agrade jugar con el sistema, obteniendo así un gusto por mover el brazo afectado y creando así nuevos caminos neuronales para el movimiento y estimulación de su motricidad fina.

Palabras Clave: Videojuego, Biomecánica, Hemiparesia, Análisis de Movimiento. 


\section{Introducción}

La hemiparesia es definida como la debilidad o parálisis parcial de un lado del cuerpo y es provocado por un daño cerebral como por ejemplo un traumatismo craneocefálico (TCE) o insuficiente oxígeno al nacer y puede adquirir trastornos motores, sensoriales, conductuales e intelectuales.[1][2] Para este proyecto se tomó en cuenta un niño de 6 años con hemiparesia izquierda, quien después de haber recibido un golpe en la cabeza al nacer produjo un coagulo de sangre lo cual originó dicha patología, el neurólogo de cabecera quien sigue con detenimiento el caso explica que esto ha ocasionado la imposibilidad en cuanto a la motricidad en las extremidades del lado izquierdo de forma parcial.

El proceso de rehabilitación depende del nivel de afectación en el sistema nervioso del paciente. Siempre que sea posible, los pacientes requieren una amplia variedad de servicios profesionales que demandan gran coordinación y esfuerzo con el fin de obtener los mejores resultados. [3][4] En el caso del paciente escogido, ha pasado por una serie de terapias para mejorar su condición física, pero en la actualidad únicamente asiste al programa de rehabilitación ocupacional donde se hace énfasis en la realización de tareas y actividades importantes en la vida diaria.

Existen diferentes técnicas en la rehabilitación ocupacional, una de ellas es el sistema "Mirror-Neuron". Esta técnica hace referencia a la estimulación del sistema nervioso en base al trabajo de aprendizaje por reflejo, el cual por medio de observación de actividades pretende que la persona afectada realice los mismos movimientos en brazos y piernas. [5][6] Tomando en cuenta este método de rehabilitación, y observando la preferencia del paciente por la tecnología digital como teléfonos celulares, tablets y televisión, se ideo la forma de que el paciente con hemiparesia, estimule el sistema motor del brazo izquierdo mediante la realización de un videojuego.[7]

El objetivo del proyecto es realizar un videojuego, adaptando un sistema electrónico en el Page brazo a un paciente con hemiparesia, para estimular la motricidad fina en su extremidad superior izquierdo ayudando a su rehabilitación. 


\section{Metodología}

La metodología de esta investigación es aplicada y como técnica la experimentación, pues es necesario incluir sistemas electrónicos que permita enviar información inalámbrica y sistemas informáticos que puedan recibirlos, también el análisis teórico en biomecánica para orientar el resultado esperado para la realización del proyecto. En los siguientes apartados se explica cómo se realiza el proyecto.

\section{Biomecánica de brazo}

De las características de la hemiparesia, se desarrolla una disminución del tono muscular en el hemicuerpo afectado [8], que para el caso de análisis es el brazo izquierdo del paciente. Otra característica en casos severos es la espasticidad que afecta a ciertas cadenas musculares como los flexores del brazo, el cual presenta un patrón típico de aducción de hombro, flexión de codo, pronación de antebrazo, flexión de muñeca y dígitos con aducción del pulgar. [8] En este caso, el paciente tendrá problemas como dolores musculares con el tiempo por falta de movilidad del brazo.

Según [9] [10], el objetivo principal de la biomecánica es evaluar la relación entre el movimiento ejecutado y el gasto de energía implicado en su realización. Para el caso del proyecto se analiza el movimiento del brazo izquierdo como se muestra en la Fig.1.
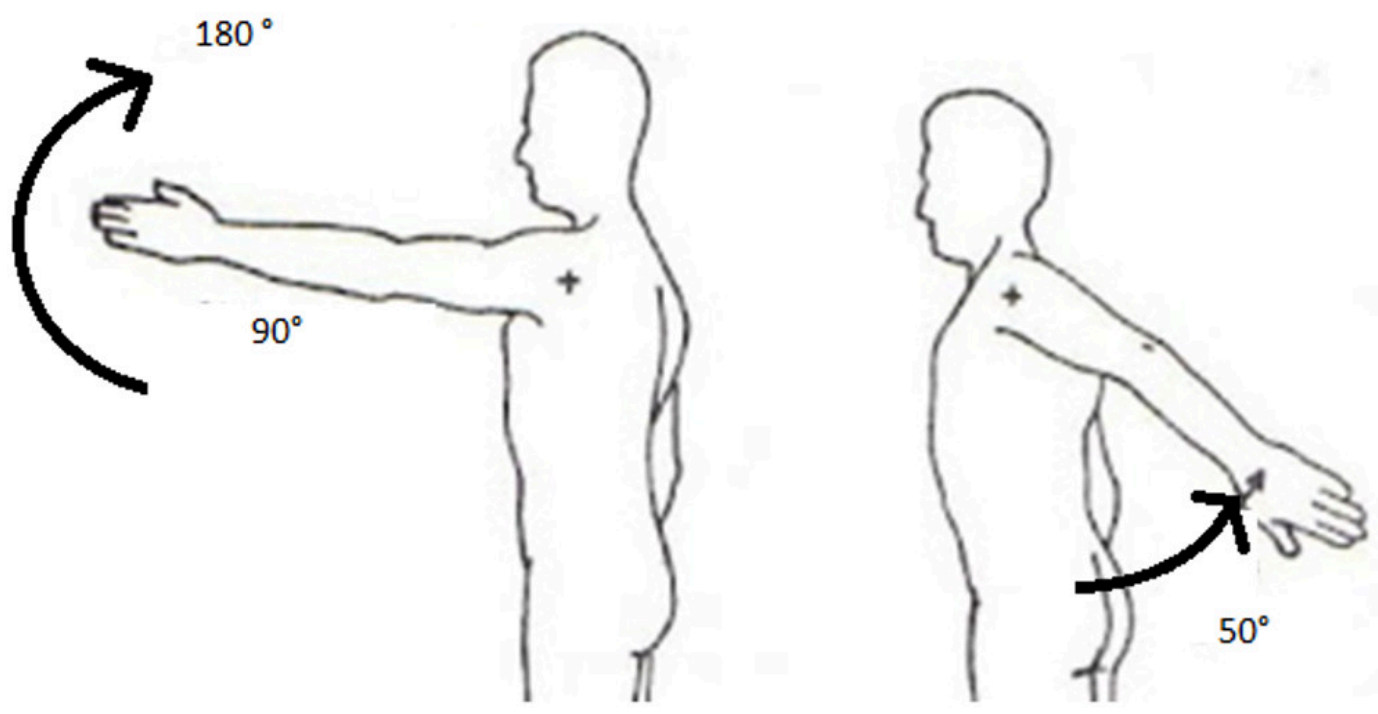

Fig. 1. Flexión- extensión del brazo izquierdo

Este movimiento se conoce como flexión-extensión, el cual se encuentra en el plano sagital, en el eje transversal y la flexión llega hasta los $180^{\circ}$ y la extensión esta entre 45 y $60^{\circ}$. [10] Sabiendo estas características se realiza un sistema electrónico adaptado a una banda elástica, que se sujeta al brazo izquierdo para que el paciente realice el movimiento de flexión de $90^{\circ}$ a $180^{\circ}$; se explica en el siguiente apartado. 


\section{Sistema electrónico}

El sistema electrónico se diseñó en base al movimiento de flexión-extensión del brazo izquierdo del paciente, y se compone de cuatro partes fundamentales como son: 1) Sensor acelerómetro; 2) Microcontrolador; 3) Bluetooth y 4) Fuente de energía, esto se muestra en la Fig. 2.

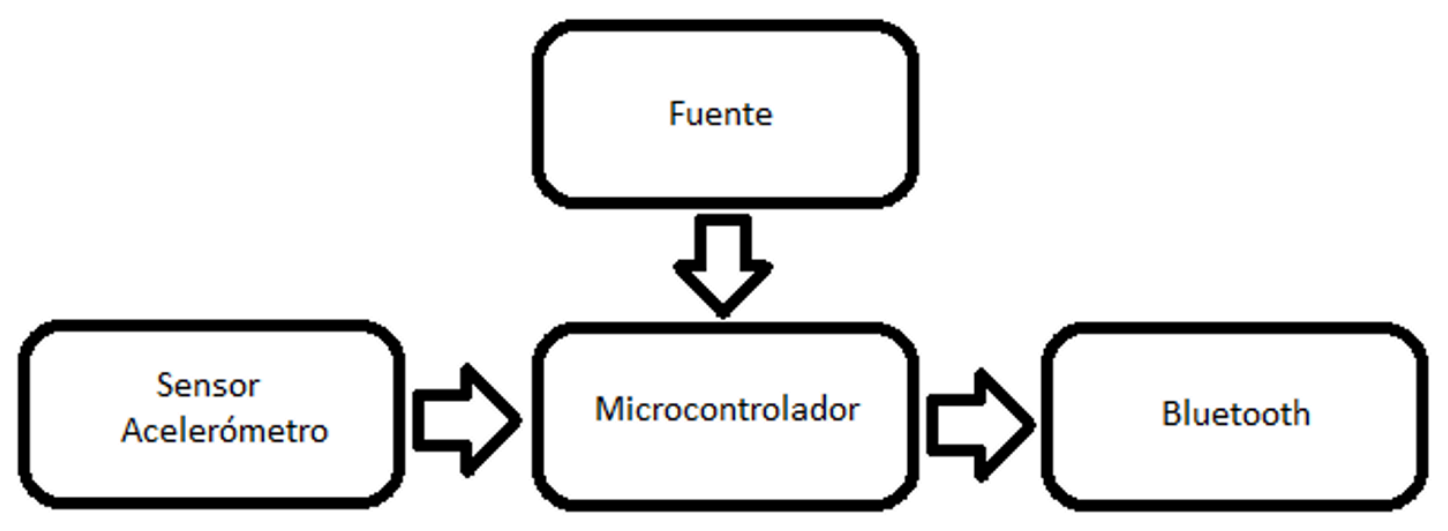

Fig. 2. Sistema electrónico adaptado al brazo izquierdo.

El sistema se encuentra basado en el movimiento del brazo izquierdo, y un elemento sensor que permite caracterizar su orientación y aceleración en ejes de tres dimensiones, el sensor acelerómetro, se muestra en la Fig.3.[11]

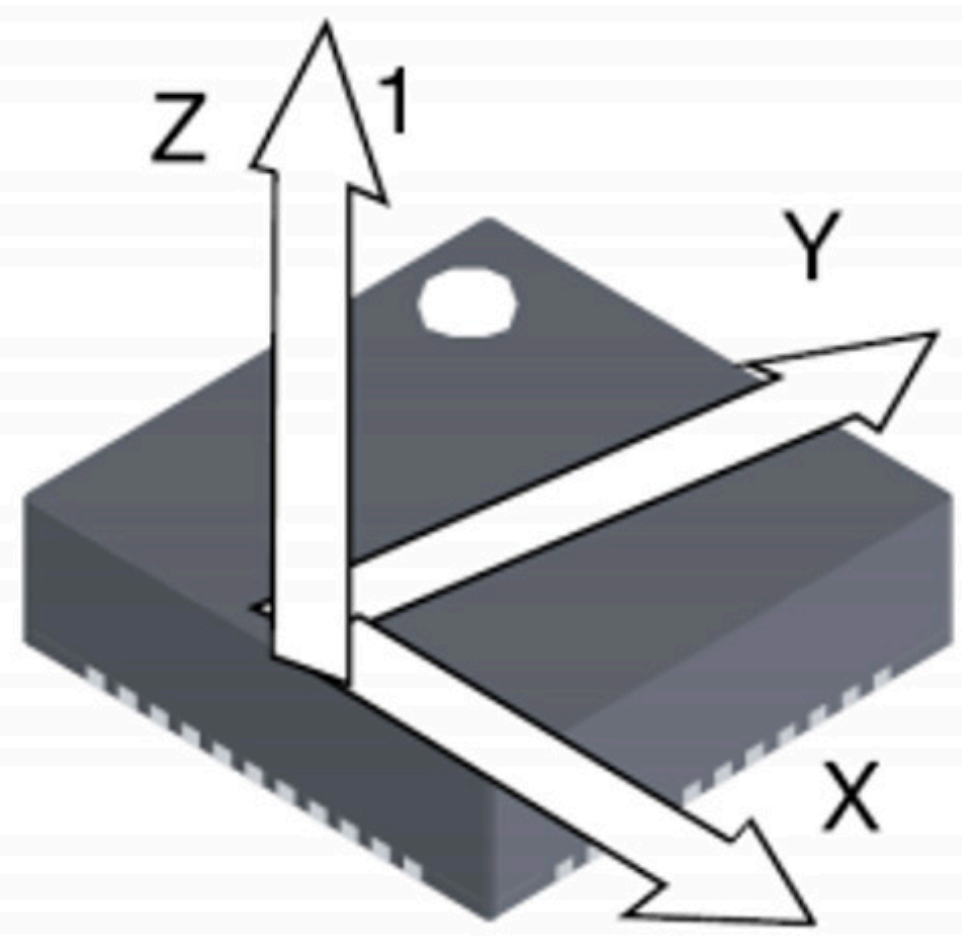

Fig. 3. Dirección de los ejes de un acelerómetro de 3 ejes. 
Gracias a la gravedad terrestre se puede usar las lecturas del acelerómetro para saber cuál es el ángulo de inclinación respecto al eje $X$ y al eje $Y[12]$; y se puede determinar su inclinación con las siguientes fórmulas:

$$
\begin{aligned}
& \text { AnguloY }=\operatorname{atan}\left(\frac{x}{\sqrt{y^{2}+z^{2}}}\right) \\
& \text { AnguloX }=\operatorname{atan}\left(\frac{y}{\sqrt{x^{2}+z^{2}}}\right)
\end{aligned}
$$

Cuando se adapta el acelerómetro al paciente, se puede conocer si al brazo izquierdo se encuentra trasladado en posición horizontal $\left(90^{\circ}\right)$ o tiene el brazo levantado $\left(180^{\circ}\right)$, esto representa el movimiento en el eje $Y$, y si el paciente rota su muñeca obtendrá valores entre $\left(-90^{\circ}\right.$ y $\left.90^{\circ}\right)$, esto representa el movimiento en el eje $X$.

El acelerómetro escogido es el MPU6050, este elemento es netamente digital y sus lecturas pueden ser adquiridas con cualquier dispositivo que tenga el protocolo de comunicación 12C[12]; para el caso del proyecto se escogió el microcontrolador Arduino Nano que tiene este recurso y se puede observar en la Fig. 4.

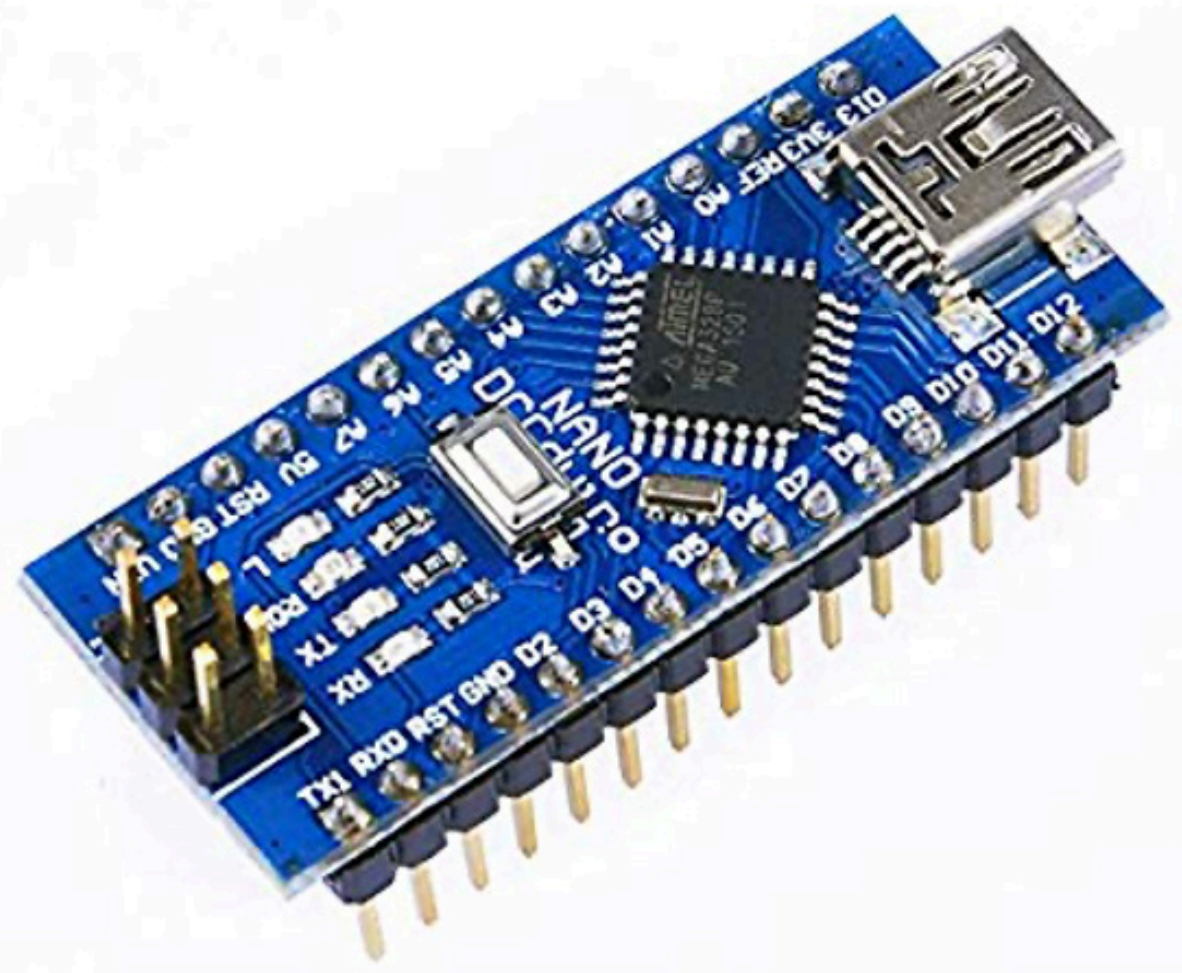

Fig. 4. Tarjeta de adquisición Arduino Nano

El proyecto se diseñó para recibir los datos del acelerómetro y que lleguen por comunicación inalámbrica a la computadora; para ello se escogió el dispositivo HC05 que es un tranceiver bluetooth que se conecta al microcontrolador retransmitiendo los datos recogidos, se puede ver en la Fig. 5. 


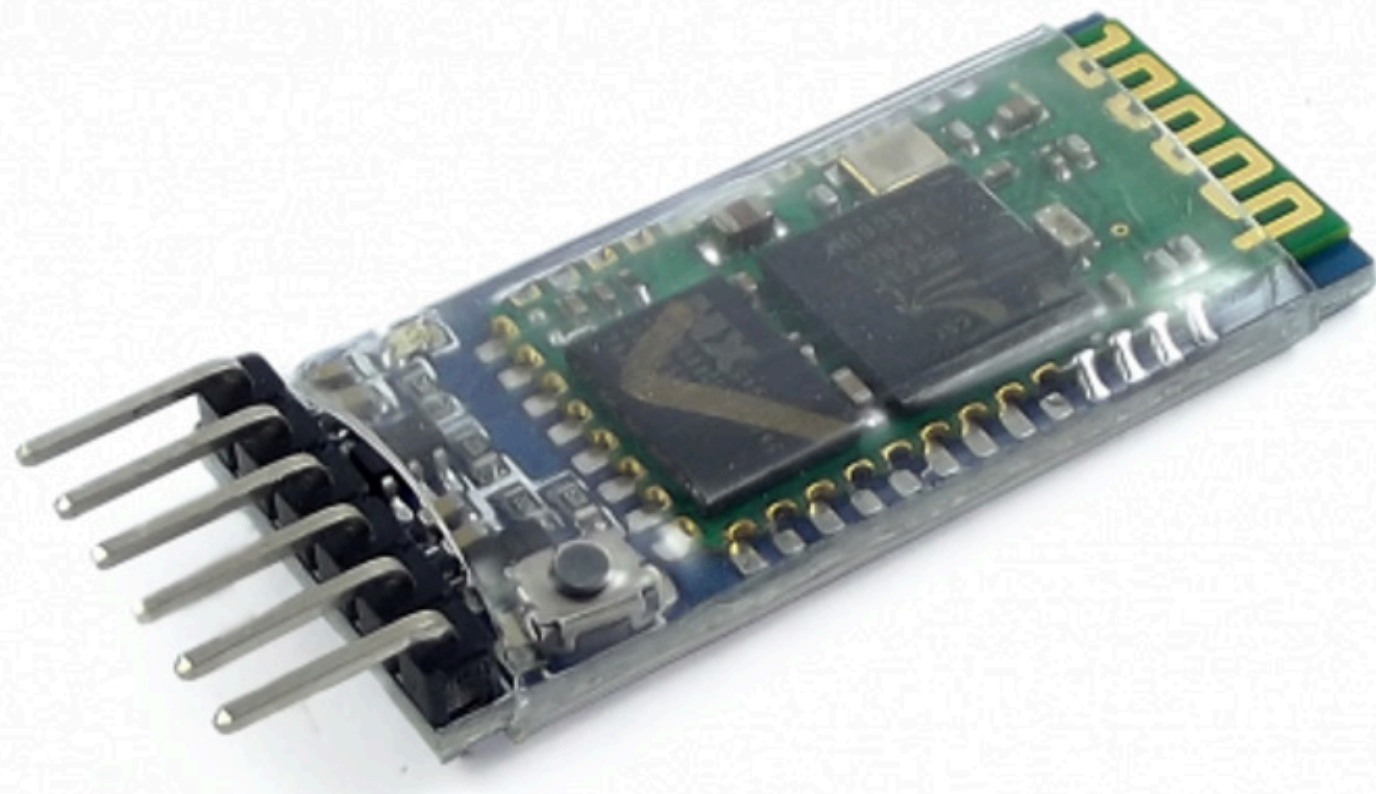

Fig. 5. Tranceiver bluetooth HCO5

Conectados los dispositivos a una batería de 5 voltios, permiten enviar la información del movimiento del brazo izquierdo del paciente hacia la computadora.

\section{Videojuegos con Unity}

La industria de los videojuegos ha demostrado un impresionante crecimiento en los últimos años y países por ejemplo como España, que es el cuarto país europeo con más consumo de este tipo de entretenimiento y el sexto a nivel mundial demuestran su interés.[7][13] Por tal motivo se escogió este tipo de plataforma ya que el entretenimiento que brinda, causa gran interés en niños y adultos.[15]

Unity es un motor de videojuegos multiplataforma, Fig.6, permite programar diferentes entornos con lenguajes de programación como Javascript, Boo y el que se usa en el proyecto C\#, y sobre todo permite una interfaz gráfica para 2 y 3 dimensiones.[14]

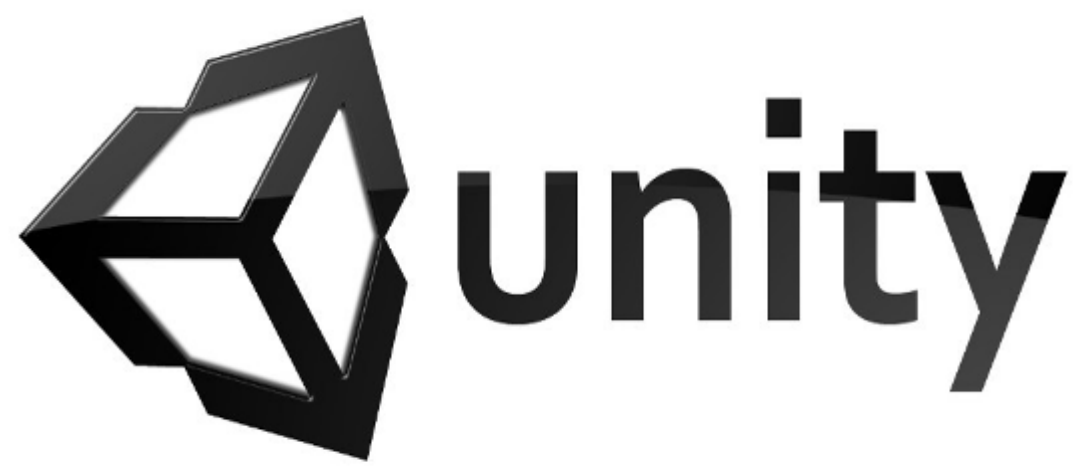

Fig. 6. Motor de videojuegos multiplataforma Unity. 
Para el diseño del videojuego adaptado al paciente, quien tiene una edad de 6 años, se creó un ambiente de fantasía en 2 dimensiones, en el cual se incorporó un jugador el cual tiene la capacidad de correr y saltar por el escenario creado, esto se muestra en la Fig. 7.

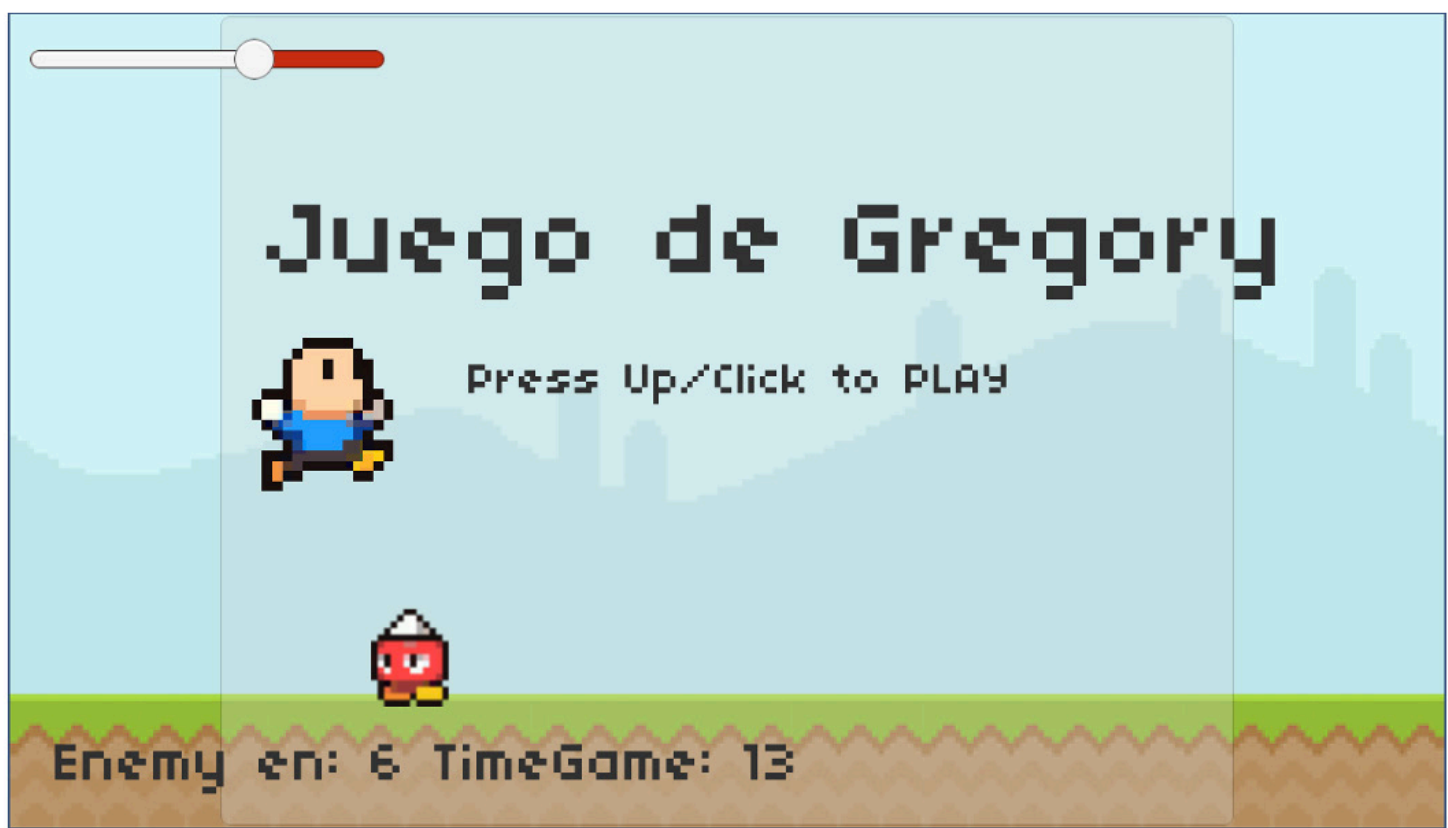

Fig. 7. Juego diseñado en Unity y adaptado al paciente.

En base a programación en C\#, se puede abrir puertos de comunicación conectados a la computadora para recibir datos, los cuales llegan a través del bluetooth que tiene la PC y la información es enviada desde la banda electrónica.

El juego tiene un único nivel, en el cual el jugador (paciente) no tiene un control remoto estándar, sino que con la banda adaptada al brazo y extendido a $90^{\circ}$ empieza a transmitir la información de posición del brazo y empieza a jugar el usuario.

Por el movimiento del brazo, el jugador puede correr más rápido o más lento, trasladando el brazo de 90 a $180^{\circ}$ y saltando cuando rota la muñeca desde 0 a $90^{\circ}$. Por consejos del neurólogo el juego debe ser controlado, para no crear síntomas secundarios que en algunos pacientes se generan como rasgos de epilepsia, máximo aconsejable 10 minutos.

\section{Resultados}

Iniciado el juego que se encuentra instalado en la computadora previamente y receptando la información del sistema de control, empieza a correr el jugador representado por un niño pequeño que debe cruzar un ambiente en movimiento.

El sistema de referencia mostrado en la Fig.8, parte del hombro del paciente, y colocando el brazo en una posición de $180^{\circ}$, marca el punto de partida a velocidad lenta de corrida. Para aumentar la velocidad del jugador se programó en el videojuego que, al cambiar la posición del brazo, en este caso levantándolo, la inclinación del sensor cambia aumentando así su velocidad. 


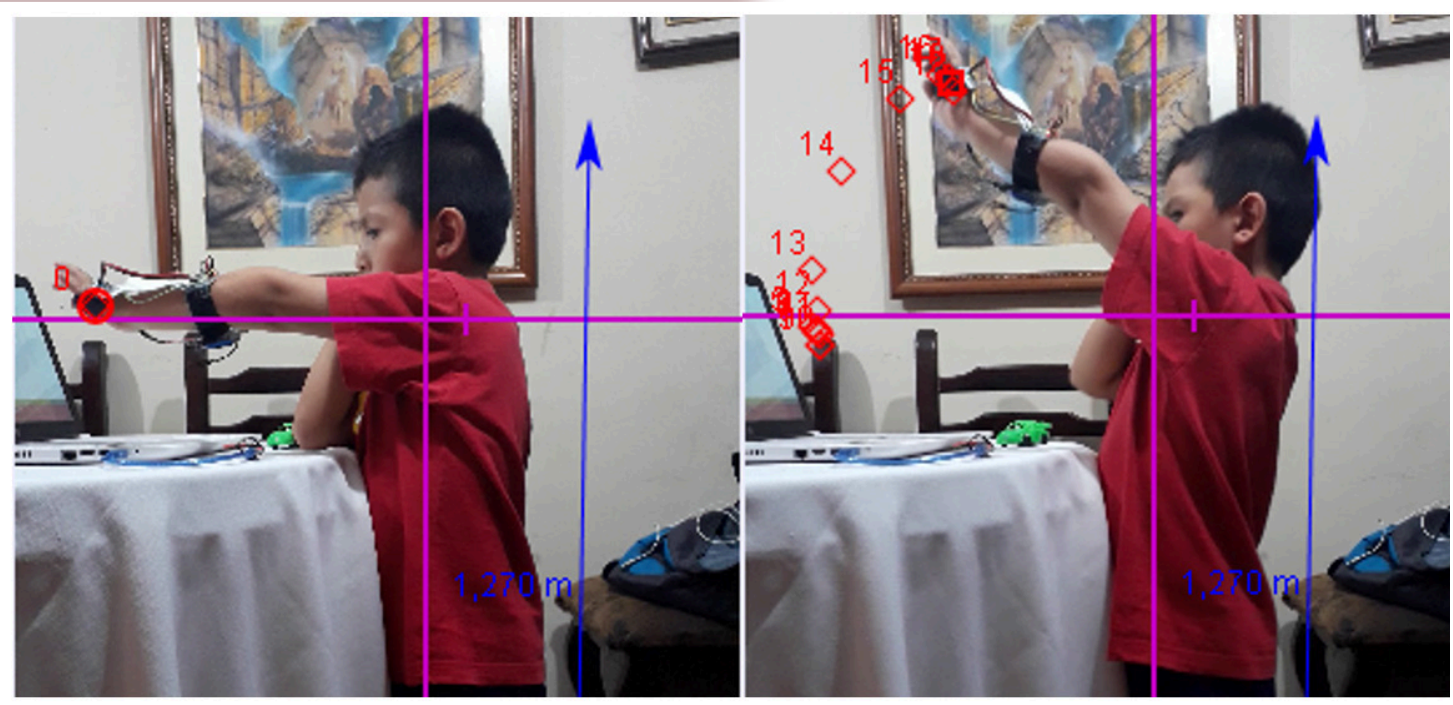

Fig. 8. Movimiento del brazo, cambiando inclinación del sensor.

El niño estimula por sí mismo la motricidad fina con el movimiento del brazo izquierdo, ya que debe colocarlo a un ángulo de inclinación que dará como resultado la velocidad en la que le guste jugar. Con el software para análisis de movimiento Tracker, permite revisar cuadro a cuadro el movimiento del brazo y se nota en la mayoría de pruebas el ángulo de desplazamiento mínimo y máximo que el paciente trabaja y se encuentra entre $130^{\circ}$ y $180^{\circ}$, y generalmente se queda jugando en un ángulo entre $160^{\circ}$ y $170^{\circ}$ como muestra la Fig. 9.
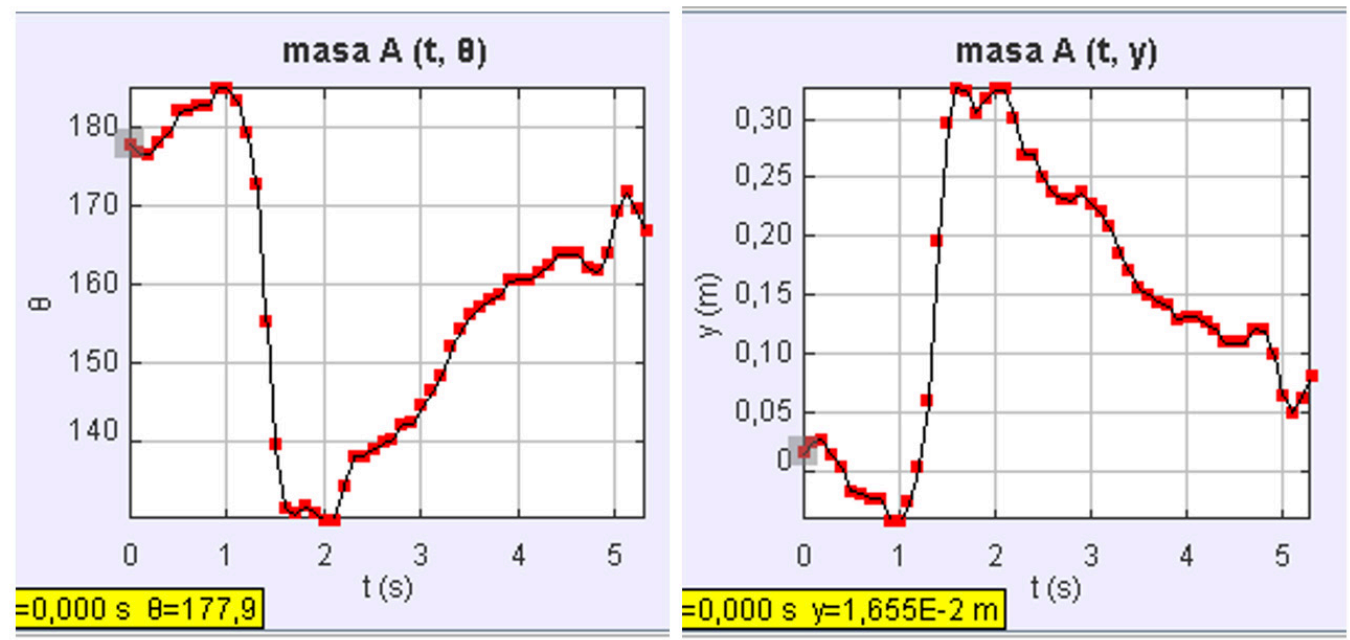

Fig. 9. Gráfica de posición angular del brazo izquierdo.

Adicionalmente, se implementó en el videojuego un personaje conocido como el enemigo, que aparece cada 10 segundos, caminando frente al jugador. El objetivo del jugador es pasar por encima del enemigo, dándole un punto por cada enemigo brincado, pero si no lo hace no recibe puntuación.

La forma de brincar del jugador fue implementada rotando la muñeca del paciente; el sensor acelerómetro capta el ángulo de inclinación al momento de rotar la mano, haciendo que este salte, esto se muestra en la Fig.10.

\section{Page




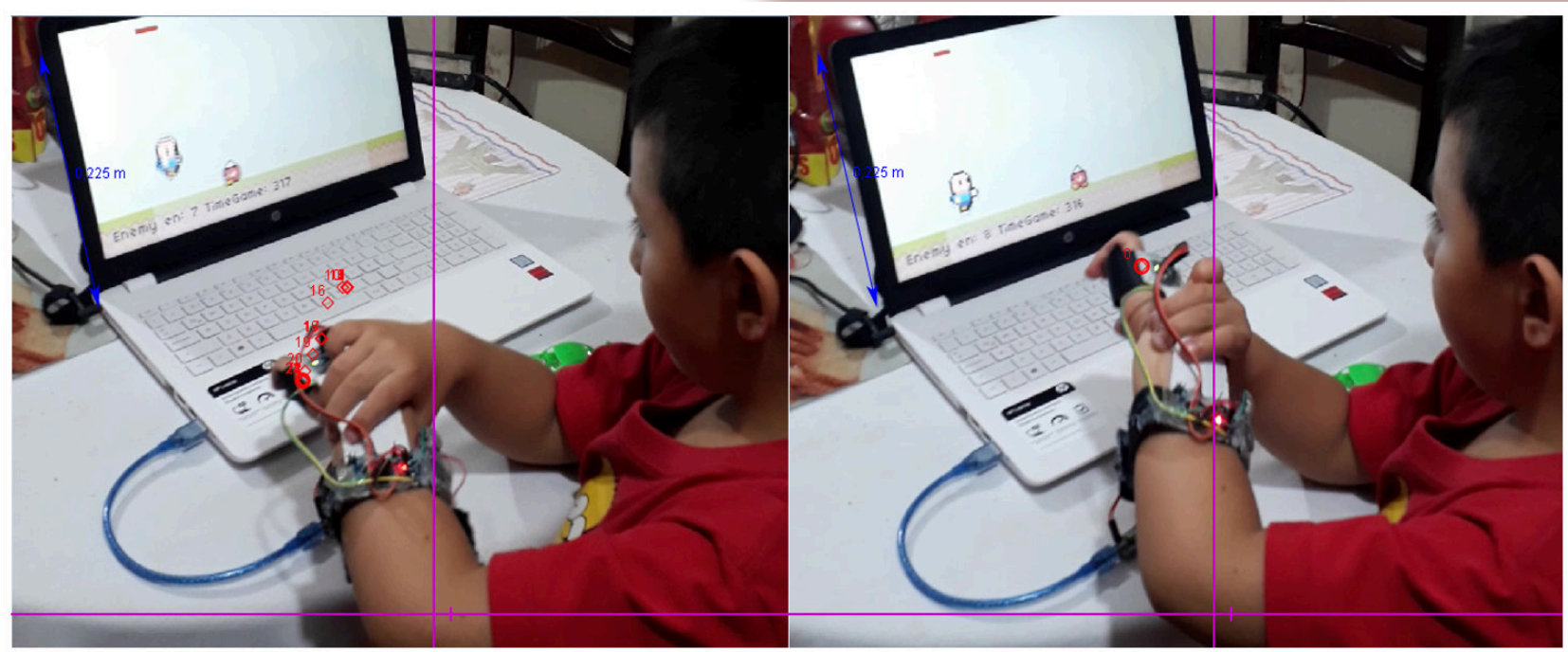

Fig. 10. Rotación de la muñeca para salto del enemigo.

Dada la dificultad del paciente porque no puede rotar directamente la mano, él se ayuda con su otra extremidad para poder realizar el giro. Fue una desventaja para el paciente, pero se espera que con más tiempo lo haga por sí mismo. Con tracker se pudo realizar el análisis de la rotación de la mano y especificar en varios experimentos el ángulo de desplazamiento de la muñeca y lo realiza entre 90 y $130^{\circ}$, según el sistema de referencia escogido, esto se muestra en la Fig.11

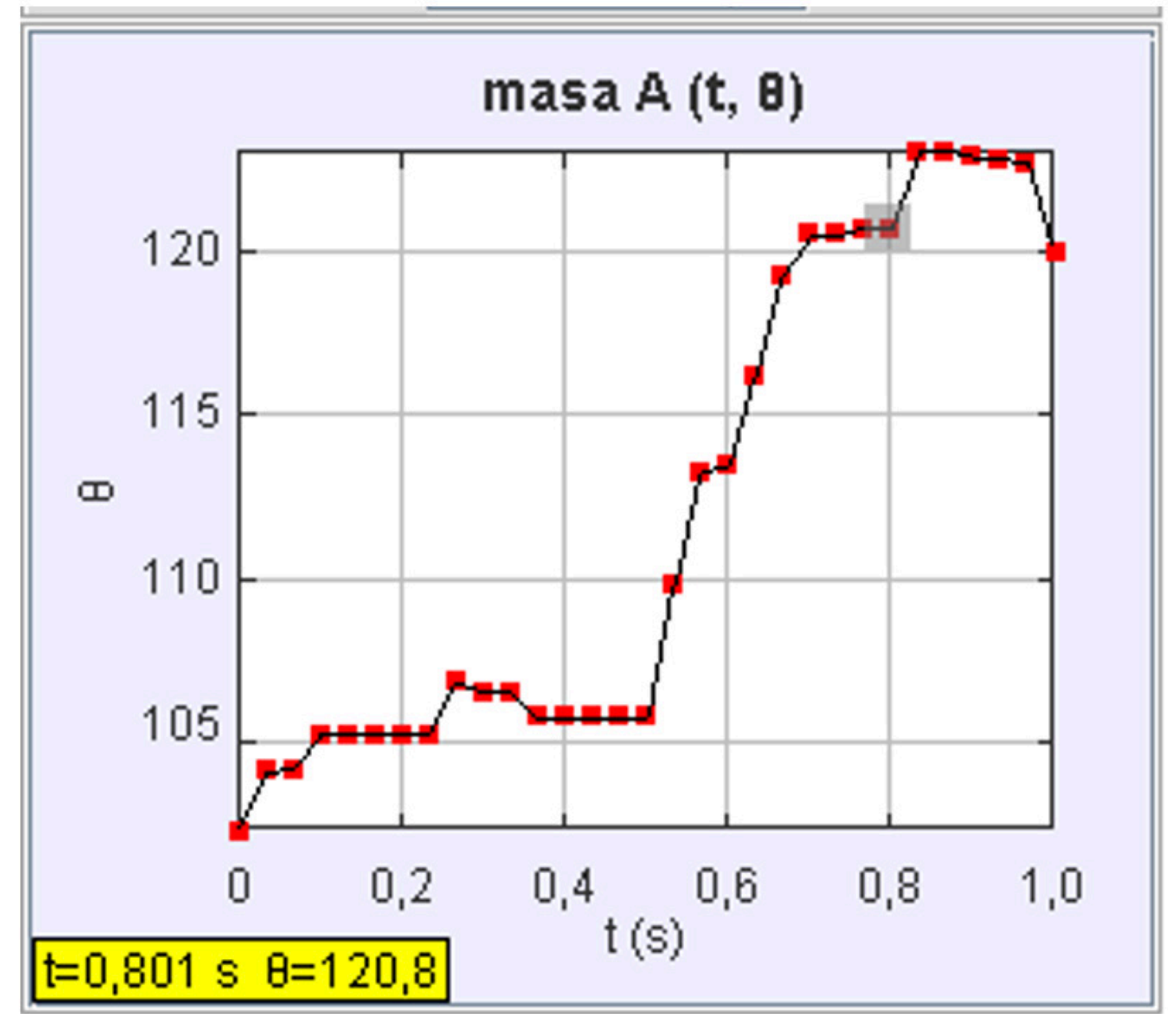

Fig. 11. Ángulo de rotación de la muñeca. 
Tomando en cuenta las recomendaciones del neurólogo de no realizar pruebas demasiadas extensas, se tomó como parámetro estimar un tiempo de prueba de 5 minutos, y como máximo 5 pruebas por día, dado este parámetro y sabiendo que el enemigo sale cada 10 segundos se toma un estimado de 30 puntos si el paciente brincara todos, la Tabla 1 muestra las pruebas en un día al azar.

Tabla 1 Pruebas de funcionamiento

\begin{tabular}{|c|c|c|c|c|}
\hline Variable & Obs & Promedio & Std. Dev. & ConteoPuntos \\
\hline P1 & 30 & .3666667 & .4901325 & 11 \\
P2 & 30 & .4 & .4982729 & 12 \\
P3 & 30 & .5333333 & .5074163 & 16 \\
P4 & 30 & .6666667 & .4794633 & 20 \\
P5 & 30 & .6333333 & .4901325 & 19 \\
\hline
\end{tabular}

Como resultado en esta serie de experimentos, se muestra que entre la prueba 4 y la prueba 5 adquiere mayor experiencia, obteniendo un mayor puntaje. Se observa el trabajo realizado por el brazo izquierdo que es el objetivo del proyecto, estimulando así su motricidad.

\section{Conclusiones y trabajos futuros}

El videojuego al ser amigable por el ambiente de fantasía incentiva el interés del paciente, haciendo que este le guste jugar con el sistema, obteniendo así un agrado por mover el brazo afectado, creando nuevos caminos neuronales para el movimiento y estimulación de su motricidad fina.

Al finalizar las pruebas, el brazo del niño terminaba cansado y se observó que la batería del sistema hacía contra peso en el miembro. Como conclusión final se puede mejorar el sistema electrónico colocando la batería no exactamente en el brazo sino en otra parte del cuerpo.

Este trabajo permite iniciar estudios con mayor profundidad en los pacientes con hemiparesia izquierda y desarrollar prototipos tecnológicos que ayuden en su rehabilitación y pueda mejorar su calidad de vida.

\section{Agradecimientos}

A todos y todas las personas que alrededor de mi familia han sabido brindar ese apoyo incondicional y a mis amigos y profesionales que me supieron incentivar en la culminación del proyecto. 


\section{Referencias}

M. Echeverría, "Hemiparesia izquierda secuela de un traumatismo craneoencefálico infantil," Repo.Uta.Edu.Ec, no. 1, p. 130, 2011.

S. Espin, "Hemiparesia izquierda a consecuencia de accidente de tránsito en adolescentes de 16 años," Repo.Uta.Edu.Ec, p. 115, 2014.

D. J. Gutierrez, D. C. A. Reyes, D. M. Tovar, D. N. Alzate, and D. F. Bohórquez, "Rehabilitacion En Trauma Craneoencefálico," Conv. ISS- ASCOFAME, 2011.

M. Jover Torres, "Rehabilitación del miembro superior mediante Terapia de movimiento inducido por restricción del lado sano en paciente neurológico.," p. 30, 2016.

T. F. Yuan and R. Hoff, "Mirror neuron system based therapy for emotional disorders," Med. Hypotheses, vol. 71, no. 5, pp. 722-726, 2010.

G. Rizzolatti and L. Craighero, "The Mirror-Neuron System," Fam. Med., vol. 48, no. 9, p. 731, 2016.

C. L. Universidad de Guadalajara, "El videojuego como herramienta educativa," Apertura, vol. 8, no. 1, pp. 136-151, 2016.

M. Murie-Fernández, M. Carmona Iragui, V. Gnanakumar, M. Meyer, N. Foley, and R. Teasell, "Hombro doloroso hemipléjico en pacientes con ictus: Causas y manejo," Neurologia, vol. 27, no. 4, pp. 234-244, 2012.

A. Repetto, Bases biomecánicas para el análisis del movimiento humano. 2007.

O. Diaz-hernandez, "Un análisis cinemático del brazo humano para biomecánica," no. September 2014, 2017.

S. Parra, "Diseño e implementacion de un sistema de control para robots bípedos," vol. 6, no. 1, Chile, 2012, pp. 1-11. 